\title{
Teaching birds to change their tune
}

Birds learn to sing in much the same way that humans learn to speak: they listen to themselves and to others, adjusting their voices until they find the right sound. By the time a bird reaches adulthood, its song usually settles into a single, stable form. Despite repeated practice, however, there are subtle differences between each rendition of the song. Until recently it was generally assumed that these variations were meaningless 'noise' that birds' nervous systems were unable to control. Now, a study by Evren C. Turner and Michael S. Brainard of the University of California at San Francisco suggests that the real reason for birds' varying tunes might be more complex.

The researchers used a computerized system to continuously monitor the song of eleven adult male Bengalese finches (Nature 450, 1240-1244; 2007). Finch song consists of a stereotyped sequence of discrete 'syllables'. The system targeted a specific syllable, delivering a burst of white noise each time a finch emitted the syllable above a certain pitch. This disrupted the bird's usual auditory 'feedback'

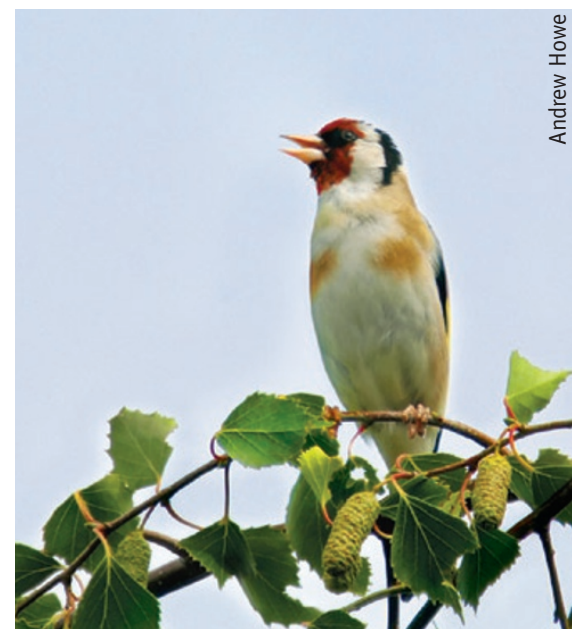

causing it to hear something other than what it expected to hear. Birds quickly learned that by adjusting the pitch of that particular syllable, they would avoid the noise and receive normal feedback. Within three days, birds sang the syllable at the 'undesirable' frequency only $17 \%$ of the time, compared with $91 \%$ at the beginning of the experiment. The researchers found that they could use this method to drive birds either to raise the pitch or to lower it. By incrementally adjusting the frequency that triggered the burst of noise, they could cause birds to substantially modify their song, producing pitches completely outside the normal range of variation. Birds' songs returned to their original range when this reinforcement was discontinued.

The birds' response indicates that changes in their song may result from a sensitive system of trial and error: a song is constantly relearned (though usually within a limited range), and birds alter vocal behavior in response to different outcomes.

Bird song is comparable to highly controlled processes in humans, such as the performance of an athlete or of a skilled musician. Such performances also differ slightly from one to the next. The team's findings imply that these variations may not be pure coincidence: humans may also be continually experimenting, finetuning each performance in response to external feedback.

\section{Karen Marron}

\section{SLEEP TN A BOTTLE?}

The effects of sleep deprivation on cognitive function may be reversed by treatment with a compound called orexin- $A$, which occurs naturally in the mammalian brain. The new research was done with rhesus monkeys and may have therapeutic implications for people who suffer from narcolepsy and other sleep disorders, as well as those whose occupations demand a high level of cognitive performance but limit or disrupt normal sleep patterns. "These findings are significant because of their potential applicability," said Samuel A. Deadwyler, who carried out the study, along with colleagues at Wake Forest University School of Medicine and the University of California at Los Angeles.

The group studied the effects of orexin-A, also called hypocretin1 , on eight adult male rhesus monkeys. The monkeys were given a cognitive test after their normal sleep cycle to determine a baseline performance level. They were then kept awake overnight for 30-36 hours and given the test again. After a 10-day break, the monkeys were again sleep-deprived for 30-36 hours and then given one of two formulations of orexin-A (either intravenous injection or nasal spray) immediately before cognitive testing.

Deadwyler's group found that the cognitive performance of sleepdeprived monkeys was significantly impaired, but treatment with either formulation of orexin-A prevented the decline in cognitive function (J. Neurosci. 27, 14239-14247; 2007). The nasal spray was more effective at improving cognitive function than was intravenous administration. Orexin-A had no effect on cognitive performance in monkeys who were not sleep-deprived. In addition, the researchers carried out noninvasive brain imaging on the monkeys during the cognitive testing. They noted that the brain activity patterns of sleep-deprived monkeys treated with orexin-A were normal, like those of monkeys who had not been sleep-deprived.

Orexin- $A$ is a known sleep-related peptide secreted by specific neurons. Once released, orexin-A can activate many areas of the brain, because its receptors are distributed in various brain regions. Orexin-A had previously been associated with narcolepsy in rats and dogs. This is the first evaluation of orexin- $A$ in primates, and its results indicate that orexin- $A$ is involved with important cognitive processes that are impaired by sleep deprivation. It seems to reactivate specific brain mechanisms rather than simply causing global arousal.

Its specific action and seeming lack of side effects suggest that orexin-A warrants further investigation for applications in treating sleep-related disorders such as narcolepsy. The protein may seem promising but has not yet been tested in humans.

Monica Harrington 